\title{
What is the impact of COVID-19 on head and neck squamous cell carcinoma patients?
}

\section{Sean Dolan ${ }^{1}$}

\section{A commentary on}

\section{Silverman D A, Lin C, Tamaki A et al.}

Respiratory and pulmonary complications in head and neck cancer patients: Evidence-based review for the COVID-19 era. Head Neck

2020; 28. DOI: 10.1002/hed.26217.

\section{Abstract}

Data sources It is not made clear in this review the different databases selected or how they conducted their search. The studies used are from 1975-2020

Study selection The authors have performed a scoping review using 84 studies ranging from 1975-2020. The majority of these are from before the 2019-2020 COVID-19 outbreak period. This is indicative of the lack of evidence on this topic and exemplifies why a scoping review was carried out rather than a systematic review. The studies that were reviewed were predominately cohort and case studies. With regards to previous treatment outcomes, a few systematic reviews were included, but again, the novel nature of this outbreak means that largely, there are only cohort or case studies available for review.

Data extraction and synthesis There are ten authors, with no indication of how many performed the literature review or if a mediator was involved in the final decision making on what papers would be reviewed.

Results Excessive consumption of alcohol, history of tobacco use, an ageing population, and comorbidities such as cardiopulmonary issues are substantial risk factors for episodes of unfavourable respiratory outcomes. The risk of these outcomes is increased by some of the toxic effects of treatments such as chemotherapy or radiotherapy. Conclusions The COVID-19 outbreak has a potentially disproportionate impact on the cohort of head and neck cancer patients, and the respiratory effects this has on these patients may increase morbidity and mortality. It is important to include alcohol and smoking cessation, along with good oral hygiene instruction in the care of these patients.

\section{Commentary}

NICE guidelines found that the majority of head and neck cancer patients have significant comorbidities and that death rates among these patients are higher than those with fewer comorbidities. ${ }^{1}$

The novel severe acute respiratory syndrome coronavirus (SARS-CoV-2) and its associated outbreak of coronavirus disease (COVID-19) has brought with it many challenges. One particular
Practice point

- Smoking and alcohol cessation advice along with good oral hygiene instruction are paramount at this time, particularly in those diagnosed with a head and neck cancer. Patients must be advised of the risks of delays in treatment and of undergoing treatment.

- Dental practices are well suited for surveillance of these patients and for stressing the importance of reducing risk factors.

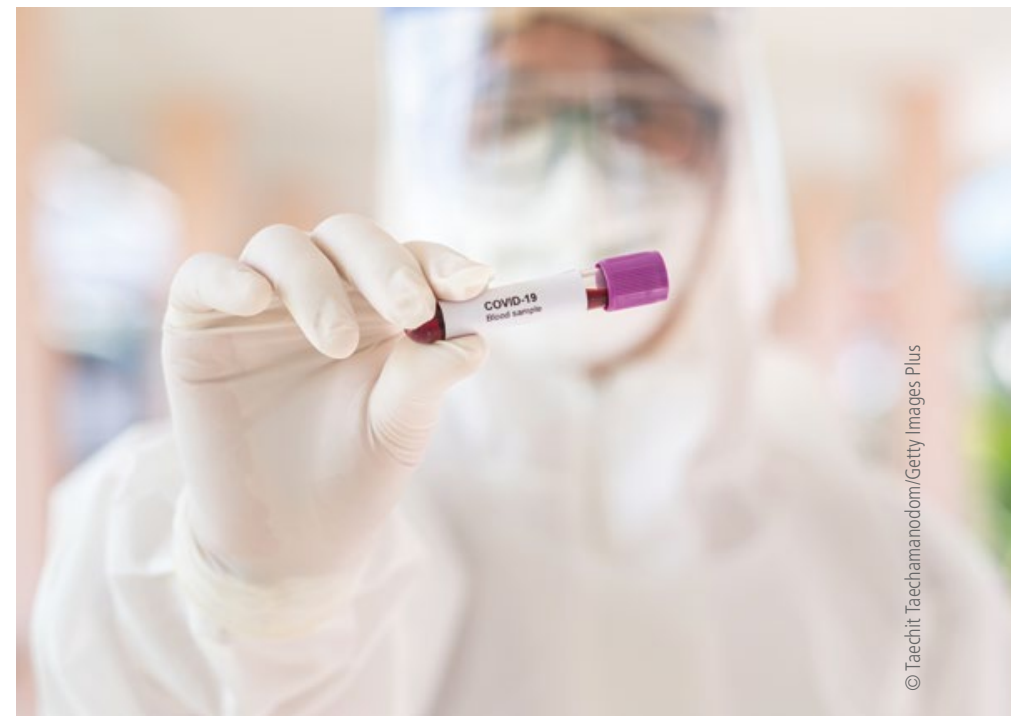

challenge, among the many, is the impact it has on head and neck squamous cell carcinoma patients (HNSCC). Studies have shown that lower respiratory tract infections increase the mortality rate in HNSCC patients. Reviews have also shown an increase in the risk of death from delayed treatment in head and neck cancer with regards to delaying radiotherapy (risk ratio (RR) 1.16, 95\% CI 1.02-1.32). ${ }^{2}$ This results in a difficult situation as treating these patients during these times may increase their risk of death due to the coronavirus, but delaying their treatment may also increase their mortality risk.

The review was a scoping review and therefore aimed to give an overview of what limited evidence there is currently on the subject; it does not focus on one type of study. ${ }^{3}$ Scoping reviews can be very useful when (as in the current situation) the research has not been expansively developed. ${ }^{4}$ It is without doubt that research in this area and on COVID-19 as a whole will continue to evolve. This paper has highlighted one key issue brought about by this pandemic. It concludes with several recommendations for the treatment of HNSCC patients during these times, taking into account the available, though limited, evidence on COVID itself and utilising the breadth of evidence published before its arrival.

There is a clear PICO question that has been followed, and although the inclusion and exclusion criteria are not made

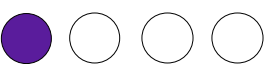




\section{SUMMARY REVIEW/COVID AND HEAD AND NECK CANCER}

clear by the authors, the review of evidence collected before the COVID-19 outbreak, which forms the background section of the paper, is extensive. The authors make it clear they are discussing HNSCC patients, particularly regarding their respiratory outcomes and the effect COVID-19 may potentially have on this. However, there is very limited evidence on the virus due to how new it is and the vast majority of the papers reviewed by the authors were published before 2019, with only 13 of the 84 references being directly related to COVID-19. Table 1 of the paper compares several studies, each with clearly outlined outcomes (the authors do state these are not necessarily the primary outcomes) which the authors of this study have managed to group together.

It is shown that there is a susceptibility to COVID among individuals with HNSCC who show increased levels of infection and there is a strong association between HNSCC and severe effects from COVID-19 infection, with the review discussing early reports from China which show a 3.5 times higher risk for any cancer patients to require ICU admission after contracting the infection. However, the reports quoted did not contain any patients with head and neck cancer.

One of the areas the paper discussed was history of smoking, and importantly it has been shown that dentists with a short behavioural intervention can have a positive effect on a patient's smoking $^{5}$ thereby reducing this risk. However, some studies have shown that dentists are not as good at assessing alcohol consumption $^{6}$ and so this area may require some improvement.
Finally, a previously published systematic review showed the link between improved oral care and a reduction in respiratory diseases. ${ }^{7}$ That review was limited to a cohort of elderly care home patients but does stress the importance of oral hygiene instructions in patients at risk of poor respiratory outcomes.

\section{Author affiliation}

DCT2, Oral and Maxillofacial Surgery, Ninewells Hospital, Dundee, UK

\section{References}

1. NICE. NICE Guidelines Improving Outcomes In Head And Neck Cancer. 2004. Available at: https://www.nice.org.uk/guidance/csg6/evidence/full-guidelinepdf-2190220525 (accessed May 2020).

2. Nouraei S A R, Middleton S E, Hudovsky A et al. A national analysis of the outcome of major head and neck cancer surgery: implications for surgeon-level data publication. Clinical Otolaryngology 2013; 38: 502-511.

3. Hanna T P, Evans G A, Booth C M. Cancer, COVID-19 and the precautionary principle: prioritising treatment during a global pandemic. Nat Rev Clin Oncol 2020; 17: $268-270$.

4. Sucharew H, Macaluso M. Methods for Research Evidence Synthesis: The Scoping Review Approach. J Hosp Med 2019; 7: 416-418.

5. Omaña-Cepeda C, Jane-Salas E, Oestrugo-Devesa A, Chimenos-Kustner E, LopezLopez J. Effectiveness of dentist's intervention in smoking cessation: A review. J Clin Exp Dent 2015; 10.4317/jced.52693.

6. Decuseara G, MacCarthy D, Menezes G. Oral cancer: knowledge, practices and opinions of dentists in Ireland. J Ir Dent Assoc 2019; 57: 209-214.

7. Sjögren $P$, Nilsson $E$, Forsell $M$, Johansson $O$, Hoogstraate J. A systematic review of the preventive effect of oral hygiene on pneumonia and respiratory tract infection in elderly people in hospitals and nursing homes: effect estimates and methodological quality of randomized controlled trials. J Am Geriatr Soc 2008; 56: 2124-2130.

Evidence-Based Dentistry (2020) 21, 52-53.

https://doi.org/ 10.1038/s41432-020-0091-9 